\title{
The CT Image Changes in Ankylosing Spondylitis from Fracture to Andersson Lesions: A Case Report and Literature Review
}

This article was published in the following Dove Press journal:

Clinical Interventions in Aging

Lu Lu Bai ${ }^{1,2, *}$
Jin Peng Du'
Xu Kai Xue ${ }^{1, *}$
Ding Jun Hao'
Wen Tao Wang'

'Department of Spine Surgery, Honghui Hospital, Xi'an Jiaotong University, Xi'an, Shaanxi 710054, People's Republic of China; ${ }^{2}$ Graduate School, Xi'an Medical University, Xi'an, Shaanxi, 7I002I,

People's Republic of China

*These authors contributed equally to this work
Background: Ankylosing spondylitis with Andersson lesions is not rare, but its potential pathogenesis and natural course remain unclear.

Case Description: We describe a case of CT image changes in ankylosing spondylitis from fracture to Andersson lesions. A 40-year-old man with a 23-year history of ankylosing spondylitis presented with acute back pain after a slight fall, and the CT showed a T12 fracture; the patient refused surgery for 12 months. The process from fracture to Andersson lesions was characterized by $\mathrm{CT}$, including the subsequent interbody bone graft with internal fixation and successful bone fusion at the last follow-up. Histopathologic analysis showed degenerative fibrocartilage tissue calcification, necrotic intervertebral disc tissue, fibrovascular hyperplasia, and focal accumulation of inflammatory cells.

Conclusion: Aseptic inflammation and persistent instability caused by a fracture contributed in the course from fracture to Andersson lesions in ankylosing spondylitis. CT can accurately track the pathological process, and interbody fusion via the posterior pedicle lateral approach can achieve satisfactory effectiveness, good fusion and kyphosis correction.

Keywords: ankylosing spondylitis, Andersson lesions, CT

\section{Introduction}

An uncommon, but well-known, inflammatory disease, ankylosing spondylitis (AS) has a prevalence between $0.1 \%$ and $1.4 \%$ globally and mainly occurs in the sacroiliac joint and spine, which results in pain, stiffness and progressive thoracolumbar kyphosis. ${ }^{1}$

As the disease progresses, ossification and calcification around the entire spine can form the "bamboo spine" ${ }^{2}$ Andersson lesions (ALs) are a complication of AS, presenting as erosive and destructive lesions of vertebral bodies and discs, which may result in sagittal imbalance and potentially spinal cord and nerve injury, ${ }^{3}$ but its pathogenesis and natural course remain unclear. In this case report, we describe a case of CT image changes in AS from fracture to ALs and review the literature to explore its potential pathogenesis, natural course, and treatment.

\section{Case Description}

A 40-year-old man was referred to our clinic with a 23-year history of AS who presented with acute back pain after a slight fall 21 months ago. On examination, the patient presented with significant percussion tenderness over the T11-T12 spine, and although the neurological examination was negative, the activity of the whole spine was
Department of Spine Surgery, Honghui Hospital, Xi'an Jiaotong University, Youyidong Road, Xi'an, Shaanxi 7I0054

People's Republic of China

Tel/Fax +86-29-87800002

Email w15686024394@I63.com
Clinical Interventions in Aging 2020:15 2227-2230

2227

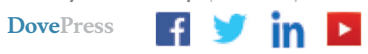

http://doi.org/| 0.2147/CIA.S282169 
restricted. CT images showed "bamboo-like changes" in the spine, and the fresh three-column fracture of T12 was characterized by no marked displacement and reduction in vertebral body height (Figure 1A). He was treated conservatively, which included a brace, rest, physiotherapy, and a regimen of intramuscular methotrexate (10 mg/week), sulfasalazine (1000 mg twice daily), and oral NSAIDS (nimesulide 0.1 $\mathrm{g}$ twice daily). However, he did not take his medicines regularly and experienced a more stooped position. The symptoms gradually worsened. Six months after the initial trauma, the patient returned to the outpatient clinic and complained of persistent low back pain with no neurological signs. CT images revealed pseudarthrosis with marked sclerosis at T12, and the upper part of the T12 vertebral body was irregularly damaged, with lesions identified as ALs (Figure 1B). We recommended surgery, but the patient refused and continued conservative treatment. Nine months after the initial trauma, the patient returned again and presented with progressive thoracolumbar pain without neurological symptoms. CT showed that the lesion involved the intervertebral space and the lower part of the T11 vertebral body, with pseudarthrosis and obvious hyperplasia (Figure 1C). Considering the history of progressive back pain, no neurological deficits, and progressive kyphosis at pseudarthrosis, we recommended surgery, but the patient still refused. Twelve months after the initial trauma, the patient was admitted to our hospital after presenting with unbearable back pain, hypoesthesia in the perineal area, walking weakness, and increases in urinary frequency and urgency. CT demonstrated that the scope of the lesions had extended, and there was severe thoracolumbar kyphosis and spinal canal stenosis (Figure 1D). The patient reported no history of tumor or
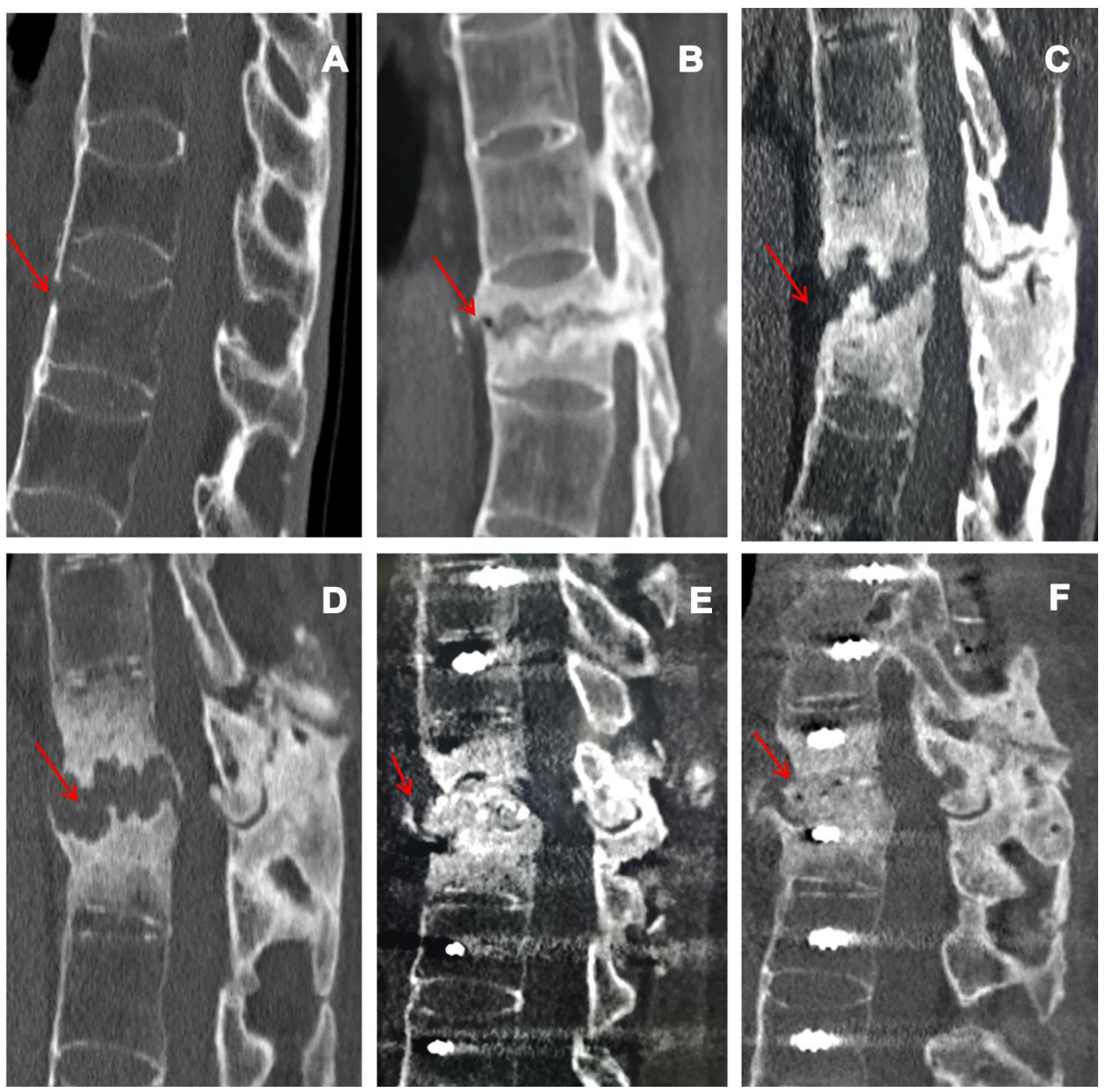

Figure I CT images showed "bamboo-like changes" in the spine, and TI2 showed a fresh three-column fracture (red arrow) (A) A pseudarthrosis with marked sclerosis at TI2, and the upper part of the TI2 vertebral body was damaged irregularly (red arrow) (B) The lesion involved the intervertebral space and the lower part of the TII vertebral body, with pseudarthrosis and obvious hyperplasia (red arrow) (C) The scope of the lesions had extended, with severe thoracolumbar kyphosis and spinal canal stenosis (red arrow) (D) The position of the pedicle screw was well fixed, the kyphosis was obviously corrected, and the intervertebral bone graft was adequate (red arrow) (E) AL segmental bone grafts have good fusion (red arrow) (F). 
tuberculosis. On examination, his legs showed weakness and numbness and hypoesthesia of the skin in the perineal area. His key muscle strength was grade IV, the deep reflexes were weakened, and the Babinski sign was negative. Laboratory examination showed that the erythrocyte sedimentation rate and C-reactive protein were $24 \mathrm{~mm} / \mathrm{h}$ and $148.4 \mathrm{mg} / \mathrm{l}$, respectively. The HLA-B27 results were positive; antinuclear antibodies and tuberculosis screening test (T-SPOT. TB) were negative; and blood counts and other serum chemicals were normal. The patient agreed to under surgery. Debridement and interbody fusion via the posterior pedicle lateral approach were performed. Postoperative CT showed great positioning of pedicle screw fixation, obvious correction of kyphosis and adequate intervertebral bone grafting (Figure 1E). Histopathologic analysis showed degenerative fibrocartilage tissue calcification, necrotic intervertebral disc tissue, and fibrovascular hyperplasia, and focal accumulation of inflammatory cells (Figure 2). When discharged, the back pain was significantly relieved, and the patient could walk independently with a brace. During the 9-month follow-up, the symptoms were significantly improved. CT showed that there was no screw loosening or fracture, no loss of kyphosis correction, and good ALs segmental bone graft fusion (Figure 1F).

\section{Discussion}

As a complication of localized vertebral or discovertebral lesions in AS patients, ALs were first described in 1937 by Andersson. ${ }^{4}$ It occurs more commonly in middle-aged males, with a prevalence of $1.5 \%$ to over $28 \% .^{5}$

The pathogenesis and natural course of ALs in AS patients is yet to be clarified. Several hypothesized causes, including inflammation and trauma, have been considered as potential etiologies. Regarding the inflammatory etiology, supporters believe that there is an absence of a previous history of trauma, characteristic of chronic aseptic inflammation on histopathologic examination, and improvements in patients' symptoms with anti-inflammatory drug treatment in the course of the disease. ${ }^{6,7}$ Conversely, in some studies, the lesions were thought to be caused by trauma because of the appearance of fracture after a minor trauma. ${ }^{8}$ Based on a completely ankylosed spine, the fracture site is the only segment of motion, and the thoracolumbar region is the site of the greatest shear/stress and the most common site of ALs. ${ }^{9}$ Chronic mechanical stress and continuous movement might hinder the healing and union of fracture, resulting in AS. In our case, CT imaging tracked the process from fracture to ALs, and histopathologic examination of the resections of the lesions showed degenerative fibrocartilage tissue calcification, necrotic intervertebral disc tissue, fibrovascular hyperplasia, and focal accumulation of inflammatory cells. Therefore, we consider that the formation of ALs in AS patients may be caused by a combination of active inflammation and mechanical factors.

In the early stage, the diagnosis of ALs may be missed or misdiagnosed on plain radiography. When there are preexisting osteoporosis, spinal changes and radiology similar to infectious spondylitis, ALs is commonly misdiagnosed as infectious spondylitis or other neoplastic diseases. ${ }^{10}$ In addition, patients usually have only mild back pain after the initial trauma, which is often ignored. Furthermore, mild spinal fractures may often be missed on plain radiography. However, CT images are more sensitive for determining the existence, location, and pathological process from fracture to ALs. ${ }^{11}$ CT images showed irregular diskovertebral osteolysis with reactive sclerosis and more frequently detected the vacuum phenomenon and paraspinal swellings. ${ }^{11}$ Thus, in patients with AS who have a past traumatic event and present mild back pain, CT should be taken into consideration when radiography shows no significant changes.

To the best of our knowledge, regarding an Anderson lesion without signs for instability, a conservative treatment
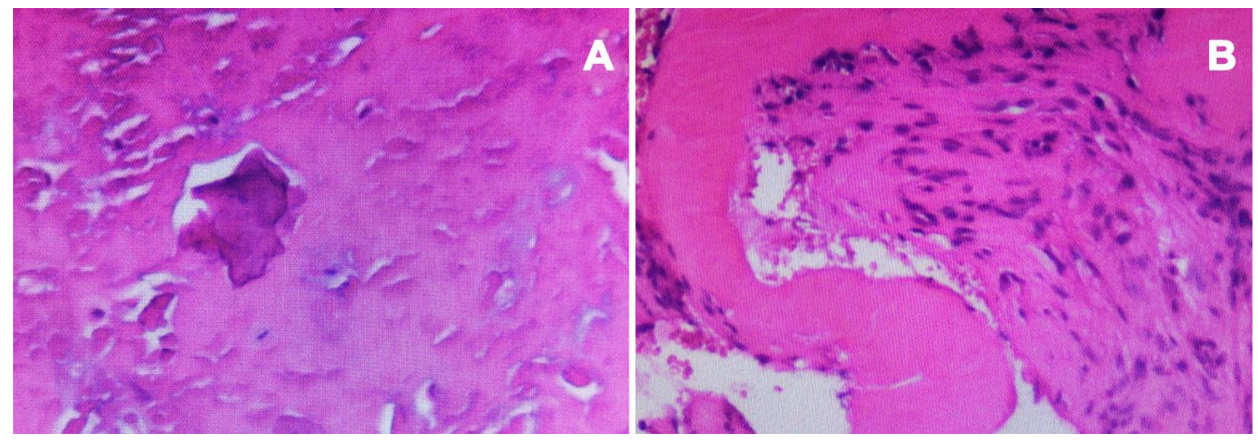

Figure 2 Histopathologic analysis showed fibrocartilage tissue calcification and degeneration, necrotic intervertebral disc tissue, fibrovascular hyperplasia, and focal aggregation of inflammatory cells ( $\mathbf{A}$ and $\mathbf{B})$. 
is initially advised. ${ }^{12}$ However, for ALs patients with severe pain, worsening symptoms, progressive kyphosis or neurological deficits, surgery is necessary. Posterior surgery can not only reconstruct the stability but also restore the sagittal balance. Zhang et $\mathrm{al}^{13}$ found that transpedicular subtraction and disc resection osteotomy can achieve satisfactory outcomes. Wang et $\mathrm{al}^{14}$ reported that a single posterior approach can remove ALs and perform bone grafts to obtain a solid fusion. In our case, debridement and interbody fusion via the posterior pedicle lateral approach were performed. During the operation, reactive osteolysis needed to be removed. At the final follow-up, satisfactory effectiveness, good fusion and kyphosis correction were achieved.

\section{Conclusions}

Aseptic inflammation and persistent instability caused by a fracture contributed in the course from fracture to Andersson lesions in ankylosing spondylitis. CT can accurately track the pathological process, and interbody fusion via the posterior pedicle lateral approach can achieve satisfactory effectiveness, good fusion and kyphosis correction.

\section{Abbreviations}

AS, ankylosing spondylitis; ALs, Andersson lesions; CT, computed tomography.

\section{Ethics Approval and Informed Consent}

This case report was approved by the ethics committee of Xi'an Honghui Hospital, and written informed consent was obtained from the patient to publish the details of his case.

\section{Acknowledgments}

This work was supported by the National Natural Science Foundation of China (grant number 81830077). Lu Lu Bai and $\mathrm{Xu}$ Kai Xue are co-first authors for this study.

\section{Disclosure}

The authors report no conflicts of interest in this work.

\section{References}

1. Dean LE, Jones GT, MacDonald AG, Downham C, Sturrock RD, Macfarlane GJ. Global prevalence of ankylosing spondylitis. Rheumatology (Oxford, England). 2014;53(4):650-657. doi:10.10 93/rheumatology/ket387

2. Maksymowych WP, Landewé R. Imaging in ankylosing spondylitis. Best Pract Res Clin Rheumatol. 2006;20(3):507-519. doi:10.1016/j. berh.2006.03.006

3. Bron JL, de Vries MK, Snieders MN, van der Horst-bruinsma IE, van Royen BJ. Discovertebral (Andersson) lesions of the spine in ankylosing spondylitis revisited. Clin Rheumatol. 2009;28(8):883-892.

4. Andersson O. Ro"ntgenbilden vid spondylarthritis ankylopoetica. Nord Med Tidskr. 1937;14:2000-2002.

5. L S, Cedoz JP, Lohse A, et al. Aseptic discitis in patients with ankylosing spondylitis: a retrospective study of 14 cases. Joint Bone Spine. 2005;72(3):248-253. doi:10.1016/j.jbspin.2004.05.015

6. S J, Baraliakos X, Listing J, et al. Persistent reduction of spinal inflammation as assessed by magnetic resonance imaging in patients with ankylosing spondylitis after 2 yrs of treatment with the anti-tumour necrosis factor agent infliximab. Rheumatology (Oxford, England). 2005;44(12):1525-1530. doi:10.1093/rheumatology/kei046

7. Hermann KGA, Althoff CE, Schneider U, et al. Spinal changes in patients with spondyloarthritis: comparison of MR imaging and radiographic appearances. Radiographics. 2005;25(3):570. doi:10.11 48/rg.253045117

8. Kim K, Lee S-H, Suk S, Lee J-H, Im Y-J. Spinal pseudarthrosis in advanced ankylosing spondylitis with sagittal plane deformity: clinical characteristics and outcome analysis. Spine. 2007;32 (15):1641-1647. doi:10.1097/BRS.0b013e318074c3ce

9. Finkelstein JA, Chapman JR, Mirza S, et al. Occult vertebral fractures in ankylosing spondylitis. Spinal Cord. 1999;37(6):444-447. doi:10.1038/sj.sc.3100837

10. Park YS, Kim JH, Ryu JA, Kim TH. The Andersson lesion in ankylosing spondylitis: distinguishing between the inflammatory and traumatic subtypes. J Bone Joint Surg Br. 2011;93(7):961-966.

11. Chan FL, Ho EK, Chau EM. Spinal pseudarthrosis complicating ankylosing spondylitis: comparison of $\mathrm{CT}$ and conventional tomography. AJR Am J Roentgenol. 1988;150(3):611-614. doi:10.22 14/ajr.150.3.611

12. Bron JL, de Vries MK, Snieders MN, et al. Discovertebral (Andersson) lesions of the spine in ankylosing spondylitis revisited. Clin Rheumatol. 2009;28(8):883-892.

13. Zhang X, Wang Y, Wu B, Hu W, Zhang Z, Wang Y. Treatment of Andersson lesion-complicating ankylosing spondylitis via transpedicular subtraction and disc resection osteotomy, a retrospective study. Eur Spine J. 2016;25(8):2587-2595. doi:10.1007/s00586-015-4213-6

14. Wang G, Sun J, Jiang Z, Cui X. The surgical treatment of Andersson lesions associated with ankylosing spondylitis. Orthopedics. 2011;34 (7):e302.
Clinical Interventions in Aging

\section{Publish your work in this journal}

Clinical Interventions in Aging is an international, peer-reviewed journal focusing on evidence-based reports on the value or lack thereof of treatments intended to prevent or delay the onset of maladaptive correlates of aging in human beings. This journal is indexed on PubMed Central, MedLine, CAS, Scopus and the Elsevier
Bibliographic databases. The manuscript management system is completely online and includes a very quick and fair peer-review system, which is all easy to use. Visit http://www.dovepress.com/ testimonials.php to read real quotes from published authors. 